\title{
Aberration Correction and Exit Wave Reconstruction Using Tilt Azimuth Data
}

\author{
A. I. Kirkland, S. Haigh \\ Department of Materials, Parks Road, Oxford OX13PH, UK.
}

Electron optical aberration correction in both TEM and STEM geometries is now been established [1,2] with a variety of imaging and probe corrected instruments installed at several laboratories around the world.

An alternative, complementary approach to direct correction involves computational compensation of measured coefficients of the wave aberration function, a posteriori during exit wave reconstruction from series of images recorded with different focus values [3], or with different illumination tilts [4]. The former extends the interpretable resolution to the axial information limit [3] and has been applied to aberration corrected images [5]. However, the latter provides information beyond the axial information limit of the microscope [4].

For corrected instruments computational reconstruction is beneficial in both geometries as it compensates for higher order aberrations, which cannot currently be directly corrected. It can also be used to locally refine the residual lower order aberrations as a function of image space. Exit wave reconstruction also gives access to complex data set not obtainable from a single image.

Data presented in this paper uses exit waves reconstructed using a Weiner restoring filter. Aberrations were initially measured globally to $5^{\text {th }}$ order from a Zemlin tableau of diffractograms and electron optically corrected to $3^{\text {rd }}$ order. Subsequently, the measured aberrations were locally refined to $5^{\text {th }}$ order using image subregions by means of a phase correlation function / phase contrast index approach [6]. Focal series datasets consisted of 16 images separated by a focal increment of $7 \mathrm{~nm}$ with the series centered about the Gaussian focus condition. Tilt series comprised of 6 short focal series (of three images) with additional axial images to assess overall focal drift taken at illumination tilts with tilt angles of $18.4 \mathrm{mrad}$. For the illumination tilts used, the samples were sufficiently thin that parallax effects could be ignored. Experimental verification of the resolution improvement using aberration corrected tilted illumination images used a specimen with a real space lattice separation beyond the axial information limit. A gold foil oriented along a $<123>$ direction satisfies this requirement

Figure 1 compares the phase and moduli of exit wavefunctions restored using both focal series and tilt defocus data sets. The only lattice detail present in the phase and modulus of the exit wavefunction (figure 1 (a) and (b)) recovered from the focal series dataset are the $\{111\}$ planes corresponding to a $0.235 \mathrm{~nm}$ lattice spacing. In contrast, the wavefunction recovered using tilted illumination data contains information from the $\{331\},\{420\}$ and $\{242\}$ reflections corresponding to lattice spacings of $0.093 \mathrm{~nm}, 0.091 \mathrm{~nm}$ and $0.083 \mathrm{~nm}$ that are beyond the $0.1 \mathrm{~nm}$ axial information limit of the microscope used 
References

[1] Haider et al., (1998) Ultramicroscopy 75, 53

[2] Batson, et al., Nature (2002) 418, 617

[3] Coene et al., (1996) Ultramicroscopy 64109

[4] Kirkland et al., (1995) Ultramicroscopy. 57355

[5] Tillmann et al., (2004) Microscopy and Microanalysis 10185

[6] Meyer et al., (2004) Ultramicroscopy 99115

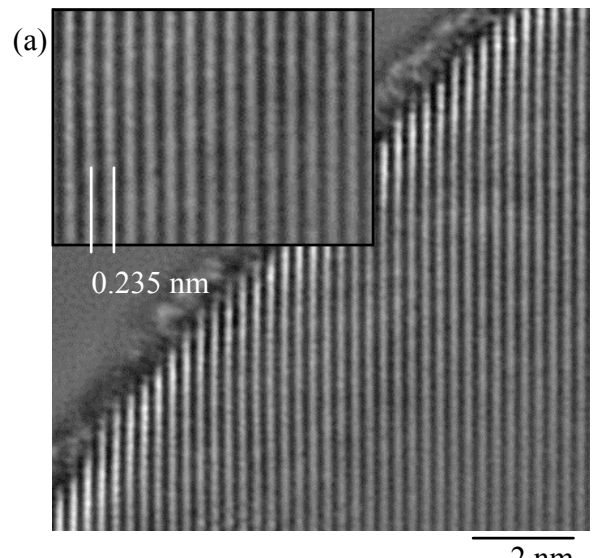

(b)

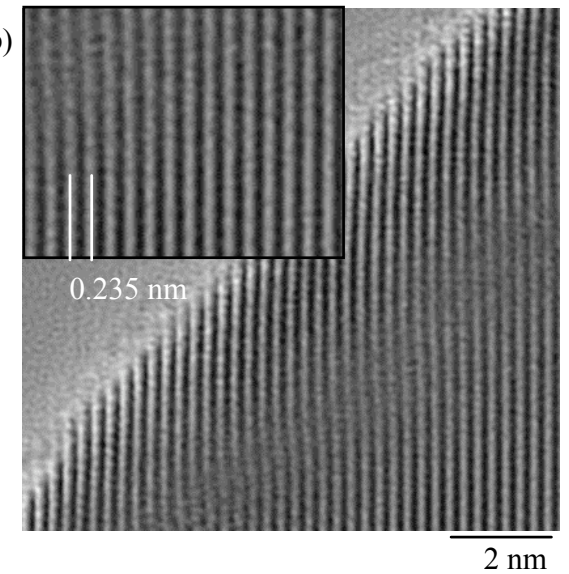

(c)

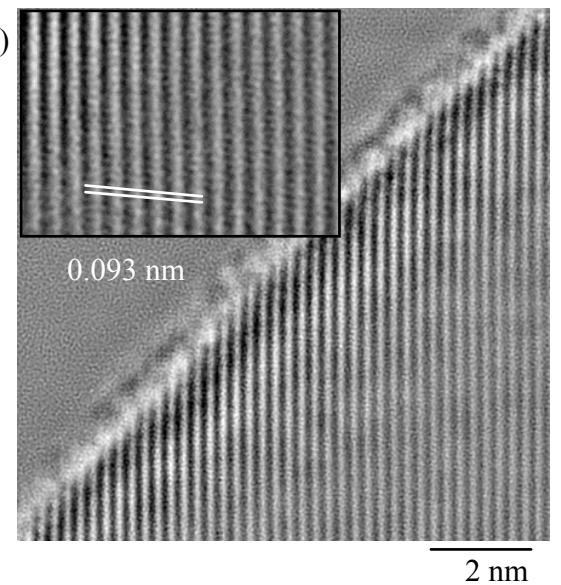

(d)

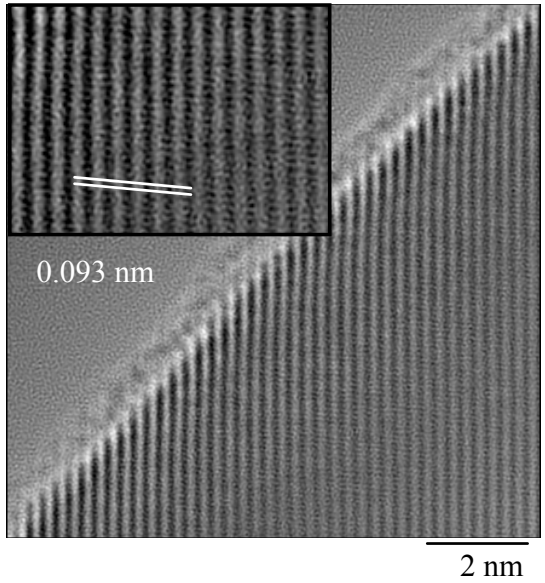

Figure 1. Exit wavefunctions recovered from a gold foil oriented along a $<123>$ direction. (a) and (b) phase and modulus restored using a 16 image focal series with a focal step of $7 \mathrm{~nm}$. (c) and (d) phase and modulus of the same specimen area restored from a 27 image tilt-defocus data set with a maximum tilt magnitude of $18.4 \mathrm{mrad}$. 